\title{
Reducing the use of antimicrobial agents in animals and man
}

On 28 M ay 1999 the Scientific Steering Committee of the European Union published a report on antimicrobial resistance [1]. The report covered all areas of antimicrobial drug use and discussed the impact on public health as well as on agricultural productivity. Considering that the problem of antimicrobial resistance is often described as complex and multifaceted, the report reaches a very clear conclusion: 'action needs to be taken promptly to reduce the overall use of antimicrobials in a balanced way in all areas: human medicine, veterinary medicine, animal production and plant protection', and it suggests that action includes: 'improved disease preventive measures, elimination of unnecessary and improper use of antimicrobials, improving the effective use of antimicrobials presently available based on more precise diagnosis of the infectious agent, and on monitoring of antimicrobial resistance and control of antimicrobial usage'.

We completely agree with the authors of the report. Enough is known to recognise the urgency of the situation, and the nature of the problem is now understood in sufficient detail to allow action to be effectively targeted.

\section{Sources of resistance}

Resistant bacteria occur in soil, water, plants, animals and fish. Resistant bacteria are probably present in all environments with which human beings are in contact, including the food we eat and the water we drink.

There is plenty of indirect evidence, and some direct evidence, that resistant bacteria or resistance genes can be exchanged between all reservoirs [1]. The challenge is to quantify the frequencies with which bacteria and genes are transmitted between the different reservoirs. This is probably extremely variable and depends on a multitude of factors. In the case of transmission of resistant bacteria from animals to man, the rate of transmission is affected by a wealth of other factors, including: how the animals are produced; how they are slaughtered; how the food is manufactured; how the meal is prepared; how frequently the foodstuff is consumed; and by whom. All of these factors vary considerably between different countries, regions, seasons, etc., making the calculation of risk estimates very difficult. However, it must be assumed, all other factors being equal, that the higher the levels of resistance in one reservoir the more frequently transmission of resistance (bacteria or genes) to other reservoirs will occur.

Selection of resistant bacteria is primarily caused by the use of antimicrobial drugs. There is a huge body of scientific evidence that the use of antibiotics is the most important factor in amplifying the level of resistance in a given reservoir. Of course, other substances with antimicrobial activity, such as detergents, may also pose a selective pressure on a resistant bacterial subpopulation, but antibiotic use is the single most important selective factor. Antibiotics are used in all reservoirs, but most antibiotics are used in food animal production and in human medicine. There are very few official data on the use of antimicrobial agents. It is estimated that nearly equal tonnage of antimicrobial agents are used in man and in agriculture worldwide [2].

\section{Spread of resistant bacteria}

Spread of resistance may be facilitated by factors other than the use of antibiotics. This phenomenon is particularly noticeable in food animal production, in which the extremely integrated breeding pyramid of food animals enables a few animal flocks to be the source of food animals worldwide. For example, a single great-grandparent hen can be the ancestor of 15 million chickens at slaughter, constituting 60 million meals. In consequence, progeny of only two-to-three great-grandparent hens could theoretically supply chicken meat to the entire Danish market. Some types of salmonellae are vertically transmissible in poultry production. Thus, antibiotic use and emergence of a resistant Salmonella clone in a single flock of greatgrandparent birds may markedly affect the levels of resistant salmonellae in the whole of Europe as well as the rest of the world. The rapid spread of the multiresistant $\mathrm{S}$. enterica serotype Typhimurium definitive type 104 on several continents probably reflects the spread of a multiresistant clone of this salmonella within the animal trade - probably pigs or cattle. The impact of the extremely integrated modern animal production process as an amplification system of 
zoonotic pathogens or resistant bacteria in food is often overlooked.

\section{Can antibiotic use be reduced?}

In a recent investigation of campylobacteriosis in the USA, it was reported that antibiotics had been prescribed to $83 \%$ of human cases of the disease [3]. In a similar study conducted in Denmark, $<10 \%$ of patients with campylobacteriosis had been prescribed antibiotics by their general practitioner [4]. The amount of antibiotic used for human beings in Spain is double that used in Denmark [5]. This is almost certainly not due to different incidences of infection. This shows that there is ample room for improvement and suggests that the amount of antimicrobial drugs used for human beings in many countries could probably be reduced by at least 50\% without appreciable risk for human health.

A gricultural use of antimicrobial agents has been much debated. A nimals raised for food production naturally should receive treatment for clinical infections. In some production systems, such as broiler chickens, treating an individual animal is impractical, so some flock medication and even prophylactic medication may be justified. However, antimicrobial drugs have increasingly been used to increase productivity. As an example, the pig production concept 'early medicated weaning' is popular in some countries. The piglets are removed from the sow at only 2 weeks of age, or even earlier. Weaning piglets at this age can take place only with the support of antibiotics to prevent disease. The system increases productivity, but it represents an unacceptable use of antibiotics because the system necessitates use that could be prevented easily by later weaning.

Antimicrobial drugs for growth promotion are administered in animal feed in lower doses than are used for therapy. This increases the growth rate of livestock and poultry under certain conditions by as much as $5-10 \%$ [6]. Consequently, this form of antibiotic use has been common practice for decades in most developed and developing countries. When the debate over the use of antimicrobial agents as growth promoters started in Denmark a few years ago, consumers demanded food produced without the use of such drugs. The Danish animal producers responded promptly by announcing a voluntary plan to suspend all use in poultry and most of the use in pigs immediately, and to phase out all use by the year 2000. The effects of the discontinuation on the levels of resistance and on productivity are being monitored. A rapid reduction in the levels of resistance to some growth promoters, e.g., glycopeptides and avilamycin, has been observed following discontinuation of their use [7]. This indirectly confirms that the high levels of resistance observed in animals and food were a consequence of the use of growth promoters. Importantly, productivity has remained essentially un- affected [7], suggesting that antimicrobial growth promoters have little, if any, effect in modern and well-managed animal production systems.

If the use of antimicrobial agents for growth promotion was suspended and production systems were improved to reduce the need for antibiotics, we believe that the amounts of the antimicrobial drugs used in agriculture today could be halved. In Denmark, agricultural use will be reduced from $>200$ tonnes annually in 1994 to c. 50 tonnes annually in the year 2000 . This reduction is taking place through a collaborative effort of industry, veterinarians and the government and without any major consequences for animal health and productivity.

Published data on the use of antimicrobial agents in fish farming (aquaculture) are very limited. Much remains to be elucidated about the purpose and type of use, as well as about the levels of resistance in bacteria from farmed fish and shellfish. The introduction of vaccines against furunculosis in fish farming in Norway has eliminated a very large proportion of the use of antimicrobial agents [1]. This is a good example of the effectiveness of vaccines, where available, in preventing disease and reducing the use of antimicrobial agents for food-producing animals.

Antimicrobial agents, such as streptomycin and tetracycline, are also used for plant protection (horticulture). The amounts used are small compared with animal use $(<0.5 \%$ of animal use in the USA [1]), but the mode of administration (spraying) and the potential effects on the environment give cause for concern. The image of fish and fresh fruit as healthy products does not fit well with antimicrobial drug use in aquaculture and horticulture.

Most problems of resistance relate to over-prescribing and the indiscriminate use of broad-spectrum antimicrobial agents. The practising veterinarian and physician often feel a greater responsibility for the individual under their care than for society at large. Therefore, solutions must aim to resolve this conflict of interest. The tools which have led to a consistently low use of antimicrobial agents in human medicine in Denmark and which have resulted in a sharp reduction in use in veterinary medicine are described below. They may serve as inspiration for others.

For more than 30 years Danish physicians have had an active antibiotic policy based on decentralised clinical microbiology services provided for all national hospitals and general practitioners. Training in prudent drug use is offered at all levels of education. The policy is supported by legislation; all antimicrobial agents are prescription-only medicines. There is no economic incitement to prescribe; narrow-spectrum antibiotics are subsidised, whereas many broad-spectrum antibiotics receive little or no subsidy. Patients are taught not to 
expect treatment with antibiotics for themselves or for their children.

As regards veterinary use, legislation ensures that the veterinarian's fee comes primarily from consultations at the farm and is independent of writing prescriptions. A ntibiotics for therapy and prophylaxis are prescription medicines sold through the pharmacy. Detailed guidelines for antibiotic use in all major animal species have been developed. These promote the use of old narrowspectrum antimicrobial agents over new, broadspectrum compounds. Some antimicrobial growth promoters have been banned because of obvious problems with cross-resistance to important antibiotics used in human medicine. A tax has been imposed on the remaining growth promoters to remove the economic benefit from their use.

\section{R esearch needs}

There is a need to compare patterns of use of antimicrobial agents in different countries to identify which factors lead to the most appropriate drug use. Furthermore, well-designed epidemiological investigations, particularly including quantitative risk assessment, are required. Such studies are expensive, timeconsuming and require a multidisciplinary approach, and they require access to valid data on the use of antimicrobial drugs. The procurement of such information is urgently needed.

Finally, surveillance of resistance is essential for the development of prescription guidelines, as well as for the establishment of a detailed understanding of the relationship between drug use and development and spread of resistance. Surveillance systems should operate in 'real time', be comparable between different laboratories and countries and include samples from all relevant sources - including animals, food and human beings. Surveillance should be supported by research on the mechanisms and the genetic background of resistance.

HENRIK C. WEGENER and NIELS FRIM ODT-M ØLLER* Danish Zoonosis Centre, Danish Veterinary Laboratory, Bülowsvej 27,

DK - 1790 Copenhagen V (e-mail: hcw@svs.dk) and *Department of Clinical M icrobiology, Statens Serum Institut, Artillerivej 5 ,

DK-2300 Copenhagen S, Denmark

\section{R eferences}

1. European Commission. Opinion of the Scientific Steering Committee on Antimicrobial Resistance. DGXXIV, Consumer Policy and Consumer Health Protection, 28 May 1999 (also available on the Internet at: http://europa.eu.int/ comm/dg24/health/sc/ssc/out50_en.html).

2. Animal Health Dossier 15: European Federation of A nimal Health. FEDESA 1997.

3. Smith KE, Besser JM, Hedberg CW et al. Quinolone-resistant Campylobacter jejuni infections in Minnesota, 1992-1998. Investigation Team. N Engl J Med 1999; 340: 1525-1532.

4. Wegener HC. The consequences for food safety of the use of fluoroquinolones in food animals. N Engl J Med 1999; 340: 1581- 1582 .

5. Frimodt-M øller N, Espersen F, Jacobsen B, Schlundt J, M eyling $A$, Wegener HC. Problems with antibiotic resistance in Spain and their relation to antibiotic use in humans elsewhere. Clin Infect Dis 1997; 25: 939-941.

6. Jensen BB. The impact of feed additives on the microbial ecology of young pigs. J Anim Feed Sci 1998; 7: 45-64.

7. Bager $F$ (ed). DANMAP 98. Consumption of antimicrobial agents and occurrence of antimicrobial resistance in bacteria from food animals, food and humans in Denmark. State Serum Institute, Danish Veterinary and Food Administration, Danish Medicines A gency and Danish Veterinary Laboratory (available on the Internet at: http://www.svs.dk/dk/z/Frm_zoo.htm). 